
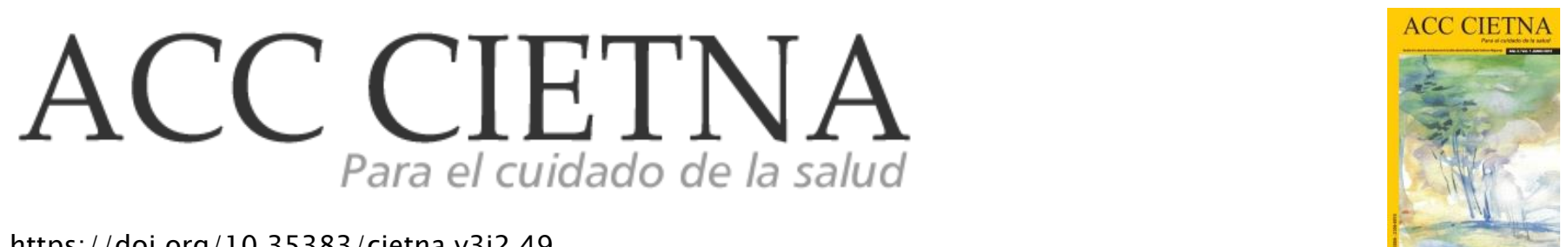

https://doi.org/10.35383/cietna.v3i2.49

\title{
La Representación Social del cuidado: Una mirada desde la perspectiva del enfermero
}

\author{
Monja Tineo Milagros Del Pilar ${ }^{1}$, Yafac Torres Ingrid Lisette ${ }^{2}$, Lázaro Alcántara Elaine ${ }^{3}$
}

INFORMACIÓN DEL ARTÍCULO
Historia del artículo:
Recibido el 14 de junio de 2016
Aceptado el 14 de octubre de 2016

Palabras claves:

Cuidado de enfermería

Enfermera

Representaciones sociales

\section{RESUMEN}

El cuidado es la esencia humana del ser enfermero; si el cuidar es lo que caracteriza las acciones de enfermería en sus diferentes dimensiones y es parte del ser cuidador, todas sus acciones se insertan en el cuidado. Este artículo presenta los resultados de un estudio cualitativo de tipo interpretativo, realizado con el objetivo de caracterizar, analizar y discutir la representación social del cuidado desde la perspectiva del enfermero. Participaron como sujetos de investigación 15 profesionales de enfermería quienes laboran en el Hospital Regional Docente "Las Mercedes"- MINSA de Chiclayo- Perú, el cual fue elegido como escenario. El referencial teórico se basó en tres conceptos: Cuidado, cuidado de enfermería y representación social, respaldado por: Waldow (1998), Kérouac (2002), Castrillón, (2003); articulados a la teoría de Representaciones Sociales de Moscovici (1979) y Jodelet (1989). Los instrumentos de recolección de datos fueron la entrevista a profundidad y el Test de Asociación libre de palabras. Las entrevistas se registraron a través de grabaciones, con previo consentimiento informado. Se siguieron criterios de rigor ético y científico. Bajo la visión de la representación social del enfermero emergieron tres categorías que forman el significado de cuidado: el acto de cuidar, estereotipos del cuidado y actividad propia de la enfermera. Se concluye el cuidado se genera a partir del pasado histórico, el reconocimiento social y la practica; generando que el quehacer

\footnotetext{
1 Licenciada en Enfermería. Enfermera del Puesto de Salud La Estancia - Olmos, Perú. Email: pilar-at@hotmail.com

2 Licenciada en Enfermería. Enfermera con trabajo independiente, Chiclayo, Perú. Email: ingrid143_3@hotmail.com

3 Doctora en Enfermería. Docente de Postgrado de la Universida Católica Santo Toribio de Mogrovejo. Enfermera asistencial del Hospital de la Fuerzas Armadas. Chiclayo, Perú. Email: elazaro@usat.edu.pe ORCID: https://orcid.org/0000-0002-7705-626X
} 
enfermero obedezcan a estereotipos que condicionan su actividad propia.

Representation Social Care: A View From the perspective of Nurse

ABSTRACT

Keywords:

Nursing Care

Nursing

Social

Representations
The approach used to carry out this research is qualitative and it builds the Social Representation of Professional Nursing Care. The location for this research was the Regional Hospital "Las Mercedes" - MINSA Chiclayo - Peru and Nursing staff who works there participated as sample research. The objectives stated are: To typify the social representation on Professional Nursing Care. Analyze and discuss the Social Representation of Professional Nursing Care. The theoretical framework was based on three concepts: care, nursing care and social representation supported by: Waldow (1998), Kerouac (2002), Castrillon, (2003), and related to the theory of social representations of Moscovi ci ( 1979) and Jodel et (1989). The instruments for gathering data were in-depth interviews and the Free Association Test words. The interviews were recorded with previous consent. Parameters were followed according to ethical and scientific rigor. Following the principles of social representation of nurses three categories constitute the meaning of care emerged: the act of caring, stereotypes care and activity of the nurse. We conclude that the nursing task joins what care and how of person-nurse interaction, which involves creating a care that uses several procedures.

\section{Introducción}

El ejercicio profesional de enfermería centrado en el cuidado, ha sido vinculado a diferentes momentos históricos, modificándose en su representación, lo que ha generado estereotipos, no solo en la sociedad, sino también en el colectivo de enfermería, condicionando sus actividades en el ámbito hospitalario.

Las dicotomías procedentes de la imprecisión para definir lo que implica el cuidado en la práctica de enfermería ha dado como resultado limitantes en su desempeño profesional, encontrando que la labor enfermera está caracterizada por actitudes que evidencian un cuidado categorizado $y$ direccionado solo a realizar procedimientos rutinarios como: administrar medicamentos, canalizar una vía periférica, tener listo los registros, informes, anotaciones de enfermería a tiempo, dejando de lado lo que la persona siente, desea o espera de su servicio.

El actuar de la enfermera para el cuidado de la persona ha generado una construcción de la imagen de enfermera deshumanizada, con poca visión para el cuidado, sin indicios de sensibilidad y empatía, aspectos primordiales para el paciente 
cuando valora el cuidado de enfermería. De este modo las enfermeras muchas veces solo realizan intervenciones de enfermería distintas de un cuidado humano. ${ }^{1}$

Respecto al cuidado que ejerce la enfermera, se afirma que su rol queda difuminado en el acto médico; encontrando que un $64 \%$ de la población asegura que dichas funciones se desarrollan bajo las órdenes del médico y estableciendo que su cuidado se limita a simples acciones, que refleja una imagen de cuidado que tiende más al aspecto técnico que profesional ${ }^{2}$. Esta realidad tal vez sea por una razón principal y es: que enfermería se ha desarrollado históricamente de manera subordinada a otras profesiones, principalmente la médica y como acción complementaria o auxiliar ${ }^{1}$, impregnado al cuidado de un significado limitante y limitado.

El significado atribuido al cuidado por los profesionales de enfermería está definido como un acto basado en conocimiento, responsable, integral, continuo y empático; que se ve influenciado por limitantes que condicionan su quehacer, entre ellas: las carencias institucionales de recursos materiales y humanos, jornadas prolongadas, turnos rotatorios, frecuentes cambios entre áreas de diferente especialidad y escasas horas de descanso; 3 diluyendo el perfil de su rol y dispersando en un sin número de tareas que la apartan del objetivo mismo de enfermería: el cuidado.

La realidad del cuidado en el escenario de estudio está caracterizado por una variedad de determinantes social es que manifiesta la permanencia de estereotipos, mitos y creencias de parte del colectivo de enfermeras que reflejan un ancoraje perpetuo de lo vocacional, sumiso, sacrificado y profesional. Esto determina que la mayoría de enfermeras identifiquen los actos de mayor responsabilidad con el campo de acción de los médicos, devaluando sus propias capacidades profesionales. Claro está que estas actitudes no están solas, sino que van acompañadas por otras dificultades de orden profesional, caracterizado por condiciones de trabajo altamente desgastantes, observándose en la mayoría de ellas una sobrecarga de actividades, reflejando un cuidado poco humanizado.

Esta realidad permite formularse una serie de interrogantes: ¿Cómo ha cambiado el significado atribuido al cuidado por las enfermeras?; ¿En realidad el enfermero se centra en el acto médico?; ¿Qué significa el cuidado para los profesionales de enfermería?; ¿Es pertinente pensar en el cuidado como simples actividades cotidianas?, ¿Cómo se representa socialmente el cuidado por el colectivo de enfermeras?. Frente a todas estas cuestiones que se piensa discutir a lo largo del estudio, se plantea la siguiente interrogante de investigación: ¿Cuál es la Representación Social del Cuidado desde la perspectiva del enfermero?

El objeto de estudio se configuró como: La Representación Social del Cuidado desde la perspectiva del enfermero. Se formularon como objetivos: Caracterizar, analizar y discutir las representaciones sociales del Cuidado desde la perspectiva del enfermero. Los resultados del presente estudio apuntaron a la comprensión de la génesis de las representaciones sociales del enfermero sobre el cuidado, partiendo de la configuración de su hacer profesional, reflejadas en las formaciones subjetivas expresadas, tal como: opiniones, actitudes, creencias y conocimientos.

\section{Metodología}

Para el desarrollo de esta investigación se consideró adecuado realizar un estudio de tipo cualitativo, que permite comprender el mundo subjetivo y a la vez interpretar el sentido y significado que los actores dan a la acción social. ${ }^{4}$ La metodología utilizada fue de Representaciones Sociales, que busca entender la dinámica de las interacciones sociales y aclarar los determinantes de las prácticas sociales; ${ }^{5}$ en este caso de cómo la 
enfermera(o) construye el significado de cuidado en su práctica profesional.

La muestra, estuvo conformada por 15 profesionales de enfermería que laboran en el Hospital Docente Las Mercedes (HRDLM) - MINSA, nivel III-1, de la cuidad de Chiclayo; respecto al tamaño, no hay criterios ni reglas firmemente establecidas, pues se determinó en base a las necesidades de información; por ello, uno de los principios que guía el muestreo es la saturación de datos.

La recolección de los datos se hizo a través de la entrevista a profundidad semiestructuradas de manera individual, las cuales fueron grabadas y transcritas en su totalidad. El análisis y tratamiento de los datos siguió el modelo propuesto por Bardín, tomado y modificado por Porto I. et.all, denominado "método de asociación de contenidos", como base para analizar e interpretar los resultados del tema en estudio: Representación Social del Cuidado desde la perspectiva del enfermero. Este análisis va más allá de las ideas manifestadas por el informante procurando extraer las relaciones encontradas entre los elementos de su discurso/texto. ${ }^{6}$

Los criterios éticos que se tomaron en cuenta, estuvo basado en los principios de la bioética personalista de Elio Sgrecia, y fueron los siguientes: principio del respeto a la vida humana, principio de libertad y responsabilidad, principio de Totalidad o Principio Terapéutico y el principio de Sociabilidad y Subsidiariedad. ${ }^{7}$

Así mismo los criterios de rigor científico utilizados en esta investigación con la que fue diseñada y desarrollada, establecido por Guba y Lincoln; fueron la credibilidad, transferencia $y$ confirmabilidad; como consecuencia de ello, logramos obtener la veracidad de los resultados. ${ }^{9}$

\section{Resultados, análisis y discusión}

Al analizar el perfil de las enfermeras del Hospital Regional Docente Las Mercedes (HRDLM), encontramos que: con relación a la edad el $46.6 \%$ de enfermeras oscila entre 41 y 50 años de edad. Es importante precisar que existe una relación directa entre la edad y el tiempo de servicio de las enfermeras en el hospital, que oscila entre 26 y 30 años de servicio (40\%).

En cuanto a la capacitación profesional alcanzada por las enfermeras de este hospital, tenemos que, el $20 \%$ de ellas no han realizado ningún estudio de postgrado, el $33.3 \%$ ha realizado una especialidad relacionada con el campo en el cual se desempeña y el $40 \%$ de las 15 enfermeras tiene el grado de maestría. Esto está relacionado sobretodo, según la referencia de las propias enfermeras a su nivel económico en base a sus ingresos y egresos, además también se justificaron de disponer de poco tiempo.

Como resultado del análisis de las entrevistas semiestructuradas a profundidad aplicada a las enfermeras, emergieron las siguientes categorías:

1. - El Acto de Cuidar: Conocimiento y complementariedad

2.-Imágenes del Cuidado: Estereotipos y Status

3.-Actividad Propia de Enfermería: Rol De La Enfermera y Limitantes del Cuidado.

1. - El Acto de Cuidar: Conocimiento y Complementariedad El acto de cuidar ha variado de acuerdo a los diferentes contextos históricos, es decir en un primer momento dicho acto solo se centraba en la forma en la que va ser ejecutado y no en el cuidado de la persona; con la introducción de los principios científicos, que eran guías norteadoras de todas las acciones de enfermería continua con el énfasis en los aspectos biológicos, pero con una preocupación en incluir aspectos psicosociales. Lo importante es que el acto de cuidado tiene ahora un respaldo científico conocimiento.9, 10 Esta categoría enfoca el cuidado como un acto dirigido a las personas, con 
una relación y un proceso que va más allá de la enfermedad; lo cual incluye comportamientos y actitudes que se demuestran en las acciones que realiza la enfermera involucrando conocimientos y ciertas características que permitan una mejor calidad de vida y bienestar.

Bajo la visión de la representación social se observa que la construcción del cuidado según las enfermeras, acontece con el conocimiento adquirido a través de la investigación y la actualización continua, aspecto que queda reconfirmado con la técnica de asociación libre de palabras, donde las enfermeras evocaron el conocimiento en un $33 \%$ asociado a la palabra "cuidado de la enfermera". Como se evidencia en el siguiente discurso:

“...El cuidado de la enfermera es un cuidado integral, de conocimientos y de muchas habilidades las cuales se van fortaleciendo a mayor experiencia..." El.

"...Yo creo que hoy el profesional de enfermería está debidamente preparado porque tiene formación académica, universitaria, científica y de investigación..." E9.

“...Es un cuidado con conocimientos, la enfermera que trabaja en este servicio tine que tener conocimientos sino no pude hacer nada..." El4.

Esto es reafirmado por Mayeroff, quien refiere que para cuidar las enfermeras necesitan disponer de un bagaje de conocimientos que permitan desarrollar la labor, permitiendo que la práctica enfermera se caracterice por una mirada integral hacia el ser humano; es decir que además de contemplar el componente biológico del individuo también se enfoque en los aspectos psicosocialesculturales y humanísticos. Por lo tanto, la práctica de enfermería va más allá del cumplimiento de múltiples tareas rutinarias, requiere de recursos intelectuales, de intuición para tomar decisiones y realizar acciones pensadas y reflexionadas, que respondan a las necesidades particulares de la persona". 11

Así mismo, el cuidado, en cuanto sistema complejo, va más allá de la simple interacción e integración de los saberes, apunta a la superación de las fronteras disciplinarias y, a la vez, refleja y amplía las discusiones acerca de las múltiples dimensiones que atañen al ser humano.12,13 Pues comprende aspectos afectivos, relativos a la actitud y compromiso, así como elementos técnicos, los cuales no pueden ser separados para otorgar cuidados, así también la identificación del significado del cuidado para quien lo otorga y para quien lo recibe, la intención y la meta que se persigue ${ }^{11}$. Por lo tanto la práctica de enfermería se ocupa más allá del acto de cuidar que une el "qué" del cuidado y el "cómo" de la interacción persona-enfermera, implica crear un cuidado que recurra a diversos procesos: la reflexión, la integración de creencias y valores, el análisis crítico, la aplicación de conocimientos, el juicio clínico, la intuición y la evaluación de la calidad de las intervenciones.

Como se evidencia en el siguiente discurso:

“...El cuidado de la enfermera es un cuidado integral, de conocimientos y de muchas habilidades las cuales se van fortaleciendo a mayor experiencia. El cuidado es humanizado por ejemplo muchas veces tenemos que ser flexibles en el horario de las visitas ya que el paciente necesita de la presencia de su familiar para darle el apoyo emocional; entonces es un cuidado integral..." El.

El concepto de integralidad engloba diferentes dimensiones del ser humano y no la enfermedad como centro de la atención, pues corresponde a una visión más amplia de las necesidades de las personas al nivel de relación entre estos y los profesionales, y a propuesta de acciones horizontalizadas y abiertas. Por lo tanto, la integralidad, así como la interdisciplinaridad, son herramientas que rompen con la práctica tradicional del cuidado en salud. Es necesario que la enfermera trabaje en estrecha colaboración con los médicos y otros profesionales de salud; el trabajo en equipo es fundamental en el cuidado, pues tanto en el individuo, o en el colectivo, se concentran numerosas acciones sanitarias que requieren interpretación, coordinación y 
articulación para que las intervenciones sean efectivas.

Finalmente se reafirma que sólo a través del fortalecimiento del conocimiento la profesión de enfermería logrará posicionarse en la sociedad, pero para ello deberá asumir la responsabilidad de actualizarse continuamente, ya que la sociedad globalizada experimenta cambios que la enfermera deberá solucionar, en pro del compromiso social asumido.

2.- Imágenes del Cuidado: Estereotipos y Status Los estudios y discusiones sobre el Cuidado se han expandido gradualmente, pues a pesar del reconocimiento que gana y el creciente interés por el cuidado, se identifican interpretaciones vagas, difusas y ambiguas que revelan las diferentes percepciones de cuidado por parte de los profesionales de enfermería. Por ello se puede afirmar que la manera como el profesional ve el cuidado, influye en su modo de pensar, de actuar, y en cómo se relaciona con su entorno. Sin embargo, no debemos dejar de lado lo que Jodelet destaca, afirmando que, las representaciones sociales deben ser analizadas en relación con los procesos de la dinámica social y de la dinámica psíquica. Entonces, según lo que propone la autora, en la construcción de una representación social debe considerarse tanto el aspecto cognitivo y psíquico, como el aspecto social del grupo al cual pertenece el individuo, ya que la interacción entre ambos afectan el origen, estructura y evolución de las representaciones. ${ }^{5}$

La construcción del cuidado de la enfermera se genera a partir del pasado histórico, el reconocimiento social y la práctica enfermera. Es importante rescatar que no es lo mismo estereotipo que representación social, tal como lo afirma Araya ${ }^{5}$ quien dice que "Los Estereotipos son categorías de atributos específicos a un grupo, que se caracterizan por su rigidez. En este sentido son más dinámicas las representaciones sociales pues éstas se modifican constantemente en la interacción diaria de las personas. Asimismo las representaciones sociales se diferencian de los estereotipos por su función: los estereotipos son el primer paso del origen de una representación...". Es decir un estereotipo es una Imagen o idea aceptada comúnmente por un grupo o sociedad con carácter inmutable.

Los profesionales de enfermería, inician la construcción del cuidado en relación a su desarrollo y valor social; así tenemos que existe un desconocimiento sobre la profesión y una invisibilidad en cuanto a su esencia por parte de la sociedad, pues no tienen bien definidas las funciones propias de enfermería, a la vez se le atribuyen valorizaciones sociales como la caridad, altruismo, abnegación, vocación y sumisión frente a otros profesionales, disminuyendo la autonomía de sus decisiones. Esto se ve reflejado en el siguiente discurso:

“...Las personas reconocen el trabajo de la enfermera como un trabajo muy dedicado, sacrificado y muy sobrecargado, generalmente lo ven como un trabajo con alto sentido vocacional.

Hay un mito que nos tiene encadenadas, el mito de la vocación que la sociedad lo une a un servilismo todavía la sociedad cree que la enfermera debe hacer su trabajo hasta en forma gratuita..." El.

En este fragmento se puede apreciar que el cuidado desde el punto de vista social es caracterizado como una labor sacrificada, dedicada con alto sentido vocacional. Estas percepciones, se han mantenido permanentes a lo largo del tiempo en la población, objetivando su cuidado profesional como una práctica que precisa de vocación, cualificándola como núcleo central a través del cual ellas prestan ayuda a los demás.

A esta situación se asocia el status y condición social que el cuidado de enfermería ha tenido y tiene una implicancia de menor prestigio profesional; esta relación surge por la posición social de la mujer y los roles atribuido a la misma ${ }^{14}$.

Cada persona cumple un rol en la sociedad lo que le asigna cierta posición más prestigiosa o no con respecto a los demás de acuerdo a cada cultura, y 
que puede variar con el tiempo. Esa posición o lugar que se ocupa en la sociedad dada por el nivel patrimonial, la ocupación laboral, el nivel cultural, entre otros es lo que se denomina status. El status siempre existe en relación con otros, o sea, dentro de la estructura social y sus enmarañadas relaciones jerárquicas; este se relaciona estrechamente con el desempeño laboral del profesional, puesto que cada uno de los status es correspondiente a sus roles. La profesión de enfermería exige un cuerpo de conocimientos como requisito indispensable para obtener status profesional; cuando analizan enfermería la ubican como semi-profesión, debido a su escaza elaboración conceptual y por la indefinición de su objeto de estudio. Situación que la torna en una actividad empírica, instrumental y operativa, que tiene dificultades para demarcar su territorio profesional, es decir no puede demostrar exclusividad en su quehacer; ${ }^{3}$ por lo que el conocimiento debe ser eje de interés de las enfermeras aunado al desarrollo de habilidades y destrezas con la que se forma la capacidad de los profesionales; solo de esta manera se podrá obtener el reconocimiento, lo que sin duda servirá de guía para la excelencia; esto es demostrado en el siguiente discurso:

\section{“...Por parte de las mismas enfermeras tienen que} seguir especializándose para tener mayor amplitud, mejorando el trabajo, porque si uno tiene mejores horizontes vas a dar una atención de calidad, integral y el beneficiado será el sector de la población..." E4.

Al aceptar la necesidad de que el ejercicio de la profesión se base en su propio conjunto de conocimientos, se reconoce la importancia de las bases conceptuales que sirven de fundamento a la experiencia profesional y ello hace meditar sobre el valor de la investigación y su extensión a estos profesionales ${ }^{15}$. El poseer conocimientos permite ser aplicado a la resolución de los problemas de la persona, desarrollar el proceso de cuidar, y califica al profesional como apto para entender ciertas actitudes y comportamientos de las personas a la vez le da autonomía al profesional para orientar sus funciones asistenciales, docentes, administrativas e investigativas, propiciando un cuidado realmente significativo.

\section{3.- Actividad Propia de Enfermería: Rol De La Enfermera y Limitantes del Cuidado.}

El cuidado como actividad propia de enfermería; es decir siendo éste la esencia de la profesión debería estar constituido por acciones transpersonales e intersubjetivas para proteger, mejorar y preservar la humanidad ayudando a la persona a hallar un significado a la enfermedad, sufrimiento, dolor y existencia y ayudar a otro a adquirir autocontrol, autoconocimiento y autocuración, 15 un trabajo que supone la organización de actividades con metas concretas y que requiere una preparación especializada, se trata de un proceso continuo de la prestación de cuidados, con el objetivo de la recuperación de la salud de la persona. ${ }^{3}$ Sin embargo las instituciones hospitalarias impregnadas aún del modelo biomédico y curativo, reduce lo humano a lo biológico alejando el trabajo de las enfermeras de su visión humanista y holística del cuidado. Lo mencionado es corroborado en el siguiente discurso:

\section{“...No se da un cuidado integral como debería de} ser, por la cantidad de paciente se dice que a nivel de MINSA debe ser de 8 a 10 pacientes por enfermera y nosotras atendemos de 23 a 25 pacientes por enfermera, en el departamento de medicina donde laboro tenemos personas de grado III de dependencia, en un 40 a 50\%..." E8.

Dichas instituciones como subsistemas sociales, cumplen la función de socializar a los individuos con pautas, normas establecidas, adaptándolos e integrándolos al sistema, de esta forma el cuidado holístico se ve influenciado por las múltiples tareas delegadas (administración del personal, de recursos materiales; alimentación de sistemas de información y tareas que no realiza otro profesional) lo que se resume en sobrecarga laboral, sumándose a ésta, ciertas limitantes como: escases de recursos de enfermería, 
desmotivación, indiferencia entre el equipo de salud, tiempo, conflicto entre enfermeras, quedando relegado acciones como la comunicación eficaz y el interactuar con el paciente y familia en forma cercana denominado por Watson "cui dado transpersonal", dichas acciones valorizadas por las personas, todo ello diluye el perfil de su rol y la dispersa en un sinnúmero de tareas que la apartan del objetivo mismo de la enfermería, ${ }^{3}$, evidenciándose en los siguientes discursos:

\section{“... Tenemos que priorizar nuestra atención porque} casi siempre estamos muy sobrecargadas (...) todos quieren atención inmediata pero el número de recursos es muy escaso, insuficiente..." El.

“(..) pienso que la atención biológica o física quedamos es más por el tiempo y por la cantidad de pacientes que tenemos, muchas veces olvidamos la parte integral, humana y pienso que eso también es un motivo para caer en la rutina". El

Son diversas las actividades que desarrolla la enfermera, el rol que cumplen dentro de las instituciones hospitalarias esta socialmente estructurado, adquiriendo las profesionales una conducta institucionalizada. La explicación de la conducta institucionalizada, estaría según Pearsons, a que la personalidad se desarrolla dentro de un sistema social y va actuar espontáneamente como pre-requisito funcional de los sistemas sociales de la que son parte, los individuos. Los individuos pasan a ser actores de estos subsistemas sociales cumpliendo un conjunto de roles y cada uno de sus roles está socialmente aceptado. Estos roles se van modelando y alienando en la interacción con otros individuos, en la cual las acciones que los actores realizan dentro de un sistema social es un modo de cumplimiento de sus propias disposiciones de necesidades y a la vez una condición para "hacer óptimas" las reacciones de los otros actores, de esta forma se puede decir que el criterio esta institucionalizado. 11 La personalidad humana es sensitiva frente a las actitudes de los otros, por lo cual su conducta va a ser premiada si está conforme con el sistema o castigada si se desvía del sistema. Lo mencionado se pone en evidencia en el siguiente discurso:

“...Recuerdo que cuando Ilegue acá a este hospital hace treinta años la enfermera era la secretaria del médico, tenía que hacerle las recetas, decía esto no me corresponde, no lo voy hacer, yo trataba de no hacerlo pero tenía que adaptarme. Si los demás lo hacían; a una la miraban mal, quedas como la pleitista, que no le gusta trabajar. Muchas veces me he peleado con muchos médicos autoritarios, pero era por imponer enfermería. Ahora de poquito a poquito nos hemos ido desprendiendo, hemos ido mejorando..." El 1.

En las instituciones de salud se espera que la función de otorgar cuidados sea a través de un trabajo vivo y que sean un núcleo de cuidado; sin embargo, el acto de cuidar es deficiente debido a que los diferentes profesionales que trabajan en los servicios de salud no realizan acciones de cuidado centrados en la persona, sino centrados en los procedimientos o en la técnica, lo que actualmente ha provocado la crisis de estas instituciones, además se agrega el hecho de que la hegemonía del modelo de acción clínica del médico empobrece y hasta anula las acciones cuidadoras del resto del equipo de salud.

En conclusión se puede afirmar que los factores enunciados, han influenciado para que la enfermería pierda la esencia como profesión de servicio a las personas; se espera que la enfermera visualice como objetivo de su trabajo, sacar a la luz su capacidad crítica y reflexiva, y de esta manera resaltar en todos los ámbitos de la salud. 32 Así mismo en que la enfermera (o) ejerza su rol profesional en forma íntegra, con ética, respetando al individuo a quien cuida y al equipo con quien interactúa, en tanto ponga la calidad como principio de su desempeño, estará manifestándose como un profesional idóneo. Eso es lo que se denomina identidad profesional. 


\section{Conclusiones}

Desde el punto de vista de la representación, se puede considerar que el cuidado desde la perspectiva del enfermero es construida socialmente, a partir de condiciones propias: valores, creencias, referentes históricos y culturales que conforman la memoria colectiva y que son compartidas. Es decir se entrelaza un conocimiento no familiar para transformarlo en otro familiar que parte de saberes e ideas ancladas sobre el cuidado, convirtiéndose en representaciones objetivadas, que explican, clasifican y evalúan el cuidado de la enfermera.

El cuidado desde la perspectiva de la enfermera se genera a partir del pasado histórico, el reconocimiento social y la práctica, a partir de ello los profesionales de enfermería, inician la construcción del cuidado en relación a su desarrollo y valor social de cada momento histórico; esto ha generado que el quehacer enfermero obedezca a estereotipos que han condicionado su actividad propia, originando formas de concebir la práctica de cuidado desde lo individual a lo social y colectivo. Estas percepciones, se han mantenido permanentes a lo largo del tiempo en la población, objetivando su cuidado profesional como una práctica que precisa de vocación, cualificándola como núcleo central a través del cual ellas prestan ayuda a los demás. Es importante precisar que por constituirse en representaciones, estos han sido y siguen siendo modificados en el que hacer enfermero, en medida que sufre la influencia propia de su contexto.

La mezcla entre estereotipos y preconceptos surgidos de la representación anclada en la sociedad, ha construido una imagen del cuidado que ha pasado por diferentes posicionamientos, de acuerdo al momento que lo caracterizaba y que ha permitido una construcción, deconstrucción y reconstrucción inacabada del cuidado enfermero. Bajo la visión de la representación social del enfermero, se objetiva que la práctica de enfermería se ocupa más allá del acto de cuidar que une el "qué" del cuidado y el "cómo" de la interacción persona-enfermera, implica crear un cuidado que recurra a diversos procesos: la reflexión, la integración de creencias y valores, el análisis crítico, la aplicación de conocimientos, el juicio clínico, la intuición y la evaluación de la calidad de las intervenciones.

Para las enfermeras el conocimiento es un pilar fundamental en su quehacer, pues se necesitan disponer de un bagaje de conocimientos que permitan desarrollar la labor, permitiendo que la práctica enfermera se caracterice por una mirada integral hacia el ser humano; es decir que además de contemplar el componente biológico del individuo también se enfoque en los aspectos psicosociales culturales y humanísticos. Por lo tanto, la práctica de enfermería va más allá del cumplimiento de múltiples tareas rutinarias, requiere de recursos intelectuales, de intuición para tomar decisiones y realizar acciones pensadas y reflexionadas, que respondan a las necesidades particulares de la persona.

\section{Bibliografía}

1. Huercanos I. El cuidado invisible, una dimensión de la profesión enfermera. [Artículo de Internet]*; 2008 febrero. [Acceso 22 setiembre de 2011 1]; 6(1). Disponible en http:/ / www.indexf.com/lascasas/documentos/lc0 510.php

2. Albert S. y Albert M. Percepción de los Usuarios y Usuarias sobre el quehacer de la Enfermera en el Primer Nivel de Atención. Redalyc. (C. Rica). 2007. 20(12): 1-13

3. Castrillón M. Dimensión Social de la práctica de la Enfermería. Edit. Universidad de Antioquia Colombia. Colombia. 2003.

4. Treviño $Z$ y Sanhueza O. Paradigmas de Investigación en Enfermería. Ciencia en Enfermería [revista en Internet]* 2005 [acceso 08 0octubre de 
2011 ; $\quad$ XI (1). Disponible en: http:/ / www.scielo.cl/pdf/cienf/v1 ln 1/art03.pd f

5. Araya S. Las representaciones sociales: ejes teóricos para su discusión. Facultad Latinoamericana de Ciencias Sociales (FLACSO), Costa Rica.2002.

6. Porto I. et. all. Método de Asociación de Contenidos: Una Alternativa para el análisis de datos textuales en las investigaciones de Enfermería. Artículo publicado en la Revista Enfermagem UERJ. Rio de janeiro, v. 11, n.3, 140p, setembro/ dezembro 2003. Río de Janeiro - Brasil.

7. Palazzi L. La Fundamentación Personalista En Bioética. [Sede Web]* Biblioteca Católica Digital [actualizada el 3 de enero de 2006; acceso 20 de Octubre de 2011]. Disponible en: http://www.mercaba.org/FICHAS/bioetica/funda mentacion_personalista_en_bioetica.htm

8. Castillo E, Vásquez M. El rigor metodológico en la investigación cualitativa [Articulo]. Vol.34 Nº4, 2003

9. Waldow V. Cuidar: expresión humanizadora de enfermería. Editorial Nueva Palabra. Brasil. 2004.
10. Grupo de cuidado. El arte y la ciencia del cuidado. Edit. Guadalupe. Colombia. 2006 11. Kérouac S, Pepin J, Ducharme F, Duquete A y Major F. El Pensamiento Enfermero. Edit. Masson, S.A. México.2002

12. Echeverria A, Vélez P. Enfermería una Profesión de Servicio. [Revista de Enf.] Actualizaciones en Enfermería. Vol. $8 \quad \mathrm{~N}^{\circ} 4$ Diciembre - Bogotá, 2005

13. Ariza C. La excelencia del Cuidado. [Revista de Enf.] Actualizaciones en Enfermería. Vol. $8 \mathrm{~N}^{\circ} 1$ Marzo- Colombia, 2005

14. Báez F, Nava V, Ramos L, Medina O. El significado de cuidado en la práctica profesional de enfermería. [Artículo de Internet]* 2008 setiembre-2009 junio [acceso 24 setiembre de 2011 ]; 9(2). Disponible en:

http://aquichan.unisabana.edu.co/index.php/aqu ichan/article/viewArticle/1476/1676

15. Artero S. Un reto para Enfermería. [Revista de Enf.] Actualizaciones en Enfermería. Vol. $8 \mathrm{~N}^{\circ} 1$ Marzo- Colombia, 2005. 